\title{
MOBILE ROBOT PATH PLANNING USING ANT COLONY OPTIMIZATION
}

\author{
T. Mohanraj ${ }^{1}$, S. Arunkumar ${ }^{2}$, M. Raghunath $^{3}$, M. Anand ${ }^{4}$ \\ ${ }^{1}$ Assistant Professor, Department of Mechatronics Engineering, Kongu Engineering College, Erode-638052 \\ ${ }^{2}$ Assistant Professor, Department of Mechatronics Engineering, Kongu Engineering College, Erode-638052 \\ ${ }^{3} P G$ Scholar, Department of Mechatronics Engineering, Kongu Engineering College, Erode-638052 \\ ${ }^{4} P G$ Scholar, Department of Mechatronics Engineering, Kongu Engineering College, Erode-638052
}

\begin{abstract}
Currently Mobile Robot has been widely used in examination and navigation particularly where static and unknown surroundings are involved. Path planning is a crucial problem in mobile robotics. Path planning of robot refers to the determination of a path, a robot takes in order to carry out the necessary task with a given set of key parameters. To find best possible path from starting point to target point, that reduces time and distance, in a given environment, avoiding collision with obstacles is a current potential research area. This paper presents SACO and ACO-MH algorithm to solve the problem of mobile robot path planning such that to reach the target station from source station without collision. The SACO and ACO-MH algorithm will give the collision free optimal path. The result obtained with ACO-MH was compared with SACO. The mobile robot environment is treated as a grid based environment in which each grid can be represented by an ordered pair of row number and column number. The mobile robot is considered as a point in the environment, to reduce the computational complexities. The ACO-MH results show better convergence speed and reduction in computational time than that of SACO through multiple MATLAB experiments.
\end{abstract}

Keywords: Mobile Robot, Path Planning, SACO, ACO-MH, Collision free and optimal path

\section{INTRODUCTION}

Mobile robot path planning is an essential issue of mobile robotics in the past two decades. Path planning is the determination of a path that a robot must take in order to pass over each point in a location in a given environment while minimizing the total cost associated with the path [1-2] and path is a plan of geometric locus of the points in a known space where the robot has to pass through. In the past two decades, various conventional methods have been developed to solve the path planning problem; some methods are cell decomposition, road map and potential field [7]. The evolution of robot global path planning algorithm from 1980 until today shows that there are numerous types of path planning algorithms proposed by researchers to solve Mobile Robot path planning problem $[11,12,16]$. These techniques show lack of alteration and a non-vigorous performance [8]. The drawbacks of potential field methods are discussed by [9]. To overcome the limitation of these approaches researchers explored to Heuristic methods. Heuristic methods have been developed progressively more over the past two decades to deal with high computational costs and complexities of classical methods, particularly for complex environments. While using heuristic algorithms, it is not sure to come across a solution, but if a solution is originated, it will be done much faster than classical methods. The main heuristic approaches employed in Mobile Robot Path Planning are Genetic Algorithm (GA), Particle Swarm Optimization (PSO), Ant Colony Optimization (ACO), Simulated Annealing (SA) and Tabu Search (TS) [6]. In case of static environment, the location of the obstacle is fixed and does not transform with time [14]. However in case of dynamic environment, the position of the obstacle changes with time $[13,16]$. In case of robot navigation, a mobile robot reaches the target destination from source station, avoiding collision with obstacles and upon iterations gives an optimal path without any human involvement. In the last decade, genetic algorithms have been extensively used to generate the optimum path by taking the advantage of its strong optimization capability [14]. Since its appearance in approximately 1992 [5], ACO has been used for solve many optimization problems such as the Travelling Salesman problem (TSP) [4], Vehicle routing, Job shop Scheduling and Flow shop Scheduling. ACO is a search technique inspired by the foraging behaviour of real ants. ACO has the ability to solve a hard combinatorial optimizations problem, the use of Ant Colony Optimization has contribute to the achievement of many investigation on Robot path planning. Any Colony Optimization algorithm is used to solve the mobile robot path planning problem in such a way that the artificial ant reaches the target point from source point avoiding obstacles [15]. A new method SACOdm to solve the navigational problem of mobile robot which is based on Simple Ant Colony Optimization Meta Heuristic (SACO-MH) was proposed in [10]. The results are optimistic for mobile robot path planning. In this study, we present an initial idea based on Simple Ant Colony optimization to select the shortest path in static known environment. The rest of this paper is organized as follows. The description of the environment and problem is described in section II, whereas section III presents the methodology of ACS and ACO-MH Algorithm in detail. 
Section IV illustrates the effectiveness of the ACS and ACO$\mathrm{MH}$ algorithm through some simulations and results. Finally, section $\mathrm{V}$ concludes the paper.

\section{PROBLEM DEFINITION}

The mobile robot environment is treated as a grid based environment in which each grid can be represented by an ordered pair of row number and column number. The environment of the mobile robot is represented in 2-D grid model. The map consists of a $20 \times 20$ square grids of identical pattern which is shown in Fig 1. The X-axis is divided equally into 20 parts and the $\mathrm{Y}$-axis is also divided into 20 equal parts. The size of grids is considered in such a manner that they can be used to accommodate obstacles of variable size and shape. The edge of each cell is of unit length. The origin or the source station $(\mathrm{S})$ of the robot is at the bottom left corner with coordinate $(1,1)$. The target station $(G)$ of the robot is at the top right corner with coordinate $(20,20)$. The mobile robot is considered as a point in the environment to reduce the computational complexities. We need to plan a path between two specified locations, a start and goal point. The path should have no collision and satisfies certain optimization criteria (shortest distance).

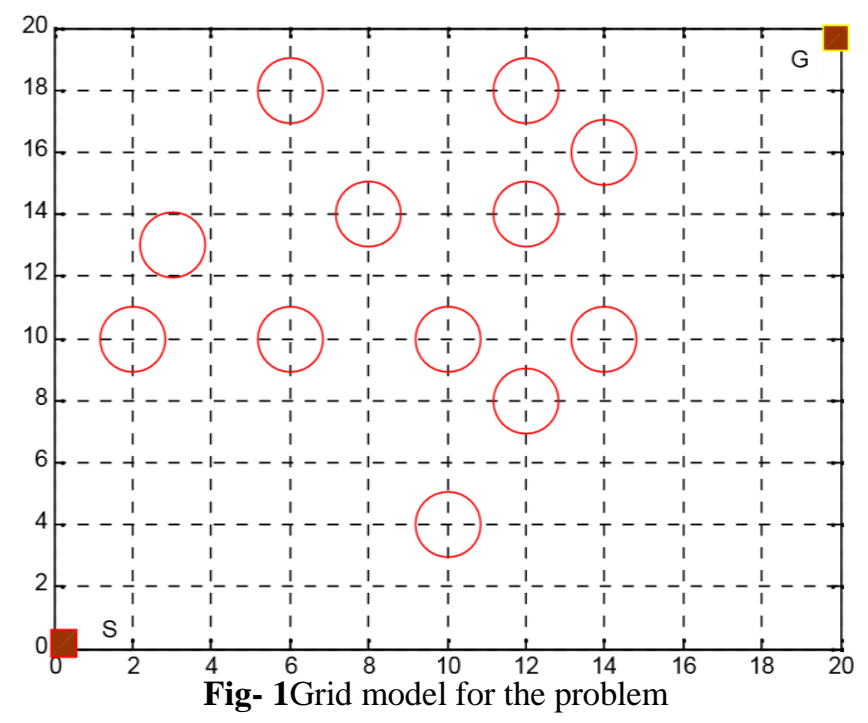

\section{METHODOLOGY}

The objective is to construct a shortest path from the source station $S$, to the target station (goal point) G, which avoids every obstacle in the map; (a path which not touch any obstacle). To solve this problem, Simple Ant Colony optimization (SACO) and Ant colony optimization MetaHeuristic (ACO-MH) Algorithm is used [5]. Ant Colony Optimization (ACO) simulates the behavior of ant colony in nature when they are foraging for food and finding the most efficient routes from their nests to food sources, it is a stochastic search algorithm, and good effect has been obtained in solving function and combination optimization, identification of various system, Path planning of mobile robots, Mining of data, network routing by using the algorithm. According to the intensity of the pheromone trail and evaporation the probability of the motion path is chosen by an ant. The probability is called as the transition probability [17]. It is also known as Ant Colony System. The following terms used in ACO.

Artificial ant: In this work simulation agents are the mobile robots that are inspired from the real ants which makes movements based on the attraction of pheromone.

Pheromone: Chemical essence deposited by an ant when walking; each ant probabilistically prefers to follow a direction rich in pheromone rather than a poorer one.

The equation for calculating the transition probability in the ant colony algorithm is following [4]:

$$
p_{i j}^{k}=\frac{\left\{\tau_{i j}\right\}^{\alpha}}{\sum_{j \varepsilon N_{i}^{k}}^{m}\left\{\tau_{i j}\right\}^{\alpha}}
$$

Where, $\tau_{\mathrm{ij}}=$ pheromone trail, $\mathrm{m}=$ No. of Ants $\alpha=$ Weight value (Positive Constant) [4]

In the equation (1) $\rho_{i j}^{k}$ represents the transition probability in which ant $\mathrm{k}$ will navigate from node $\mathrm{i}$ to node $\mathrm{j}$

The numerator on the right side of the equation represents the intensity of the pheromone trail $\left\{\tau_{\mathrm{ij}}\right\}$ between nodes $i$ and $j$ with a corresponding weight value of $\alpha$. The denominator on the right side of the equation is a summation of the products of the pheromone intensity for all possible moving paths [14].

Travelling paths have been constructed to be defined by all ants. The pheromone trails are updated in all iteration. This is done by first evaporative the pheromone value on all paths by a constant factor, and adds pheromone on the paths. Pheromone evaporation is implemented by [3].

$$
\tau_{\mathrm{ij}}=(1-\rho)^{*} \tau_{\mathrm{ij}}
$$

Where $0<\rho \leq 1$ is the pheromone evaporation rate. The parameter $\rho$ is utilized to avoid limitless accumulation of the pheromone trails and it enables the algorithm to "forget" bad decision previously taken [14]. After evaporation all ants deposit pheromone on the paths they have crossed in the motion paths. The change of the pheromone is following [5]:

$$
\tau_{\mathrm{ij}}=\tau_{\mathrm{ij}}+\sum_{k=1}^{m} \Delta \tau_{\mathrm{ij}}
$$

Where, $\Delta \tau_{\mathrm{ij}}$ is the amount of pheromone ant $\mathrm{k}$ deposits on the paths it has visited. It is given as:

$$
\tau_{\mathrm{ij}}=\frac{1}{C^{k}}
$$


Where

$\mathrm{C}^{\mathrm{k}}$, the length of the path build by the $\mathrm{k}^{\text {th }}$ ant.

\subsection{Pseudo Code of ACS Algorithm for Mobile}

\section{Robot Path Planning}

The Pseudo Code sequence of ACS is given below [18].

Represent the solution space by constructing a grid map

Locate the static obstacles, start and goal point

Initialize ACO parameters

If iteration (i) $=1,2,3,4,5,6, \ldots \ldots \ldots \ldots \ldots \ldots+n$

Else if ant $(\mathrm{m})=1,2,3,4,5,6, \ldots \ldots \ldots \ldots \ldots+\mathrm{n}$

Else if nodes $(n)=1,2,3,4, \ldots \ldots \ldots \ldots \ldots+n$

Compute the probability of the $\mathrm{m}^{\text {th }}$ ant next nodes

Move to the next nodes by computed probability

Store history of past location of nodes in an array

If current location of nodes is equal to destination

Break the nodes (n) loop

End

End

Evaluate fitness and store path distance of $\mathrm{m}^{\text {th }}$ ant

Compute pheromone amount generated by $\mathrm{m}^{\text {th }}$ ant

End

Update pheromone amount of the entire map

End

\section{SIMULATION RESULTS AND DISCUSSION}

The result of applying the Ant Colony Algorithm (ACS and ACO-MH Algorithm) is presented in this section. In order to make objective conditions three experiments were conducted with different test conditions. Simple experiments are conducted with varying the number of ants. All simulations were done with MATLAB R2009 (b). The parameter specifications are shown in Table 1. For all the experiments the pheromone evaporation rate $(\rho)$ is fixed as 0.10 .

\subsection{Experiment 1}

In this experiment the obstacles are located in random manner for obtain the optimal path. The Figures 2 and 3 shows the results of SACO and ACO-MH algorithms. The feasible path cost $31.799 \mathrm{~cm}$ was found for SACO and for ACO-MH $28.6274 \mathrm{~cm}$ was obtained which has the minimum path cost compare to the SACO algorithm. While increasing the number of ants the algorithm founds the optimal path. If the number of ants increases beyond certain level there is no considerable change in the path distance.

Table 1 Parameter Specification for ACO algorithm

\begin{tabular}{|l|l|l|}
\hline Parameters & SACO & ACO-MH \\
\hline No. of Ants $(\mathrm{m})$ & 10 & 10 \\
\hline Weight value $(\alpha)$ & 0.25 & 0.25 \\
\hline Heuristic Factor $(\beta)$ & 0 & 1 \\
\hline Radius of the obstacles & 1 & 1 \\
\hline $\begin{array}{l}\text { pheromone evaporation } \\
\text { rate }(\rho)\end{array}$ & 0.10 & 0.10 \\
\hline
\end{tabular}

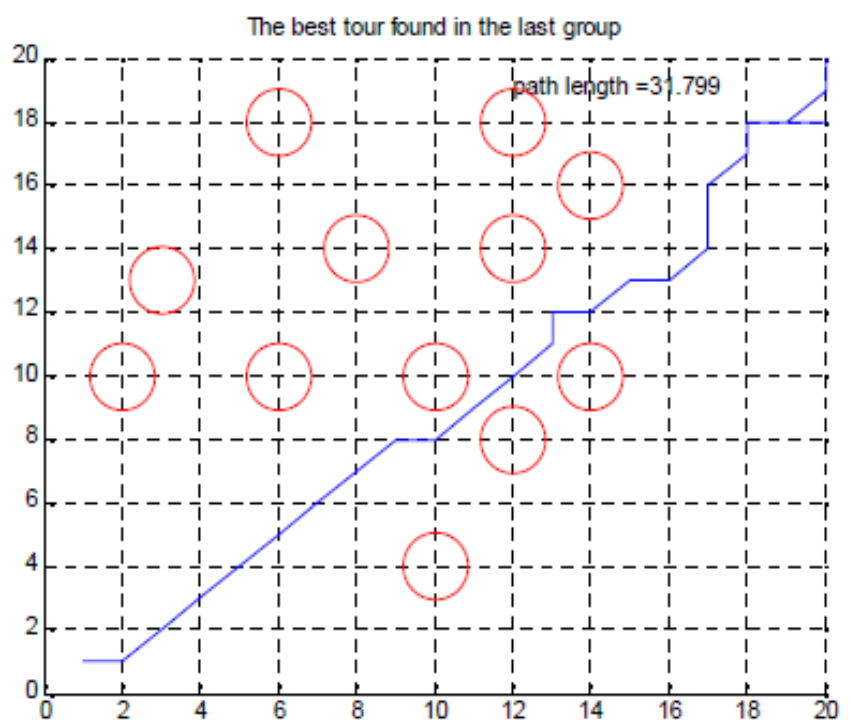

Fig- 2 Optimal Path found with SACO for Experiment 1

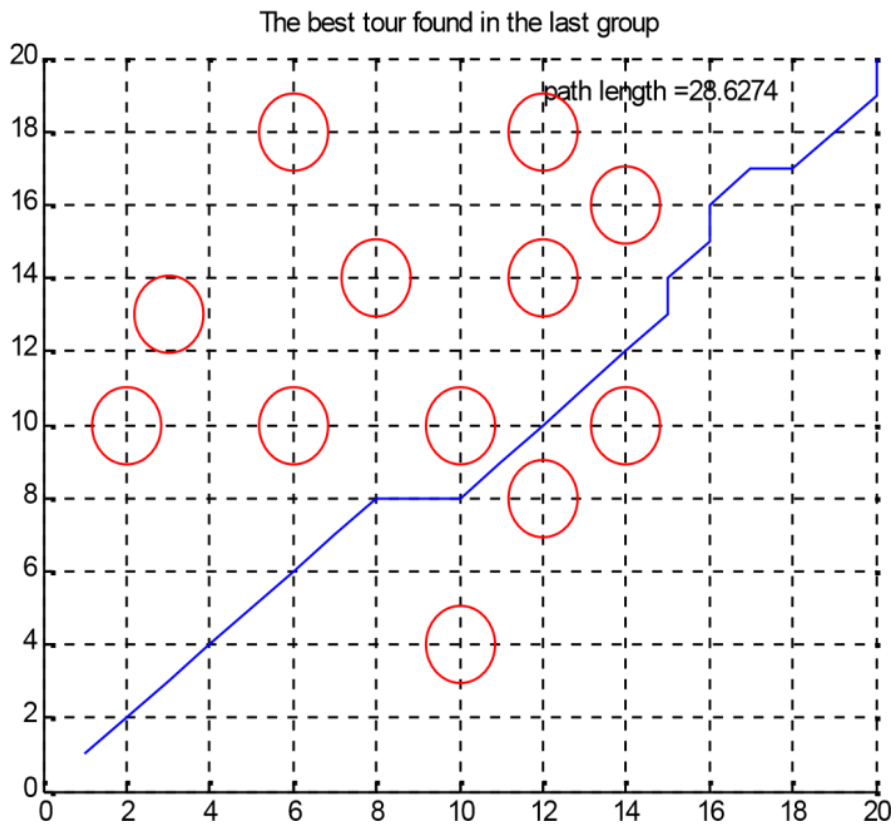

Fig- 3 Optimal Path found with ACO-MH for Experiment1 


\subsection{Experiment 2}

In this experiment the obstacles are located in vertical line to block optimal diagonal path. The Figure 4 and 5 shows the optimal results of SACO and ACO-MH algorithms. The optimal path found with ACO-MH is $32.1421 \mathrm{~cm}$ but in the case of SACO it is found that $33.8995 \mathrm{~cm}$. Both of the algorithms are unable to follow the diagonal path because of the obstacles location on the grid map.

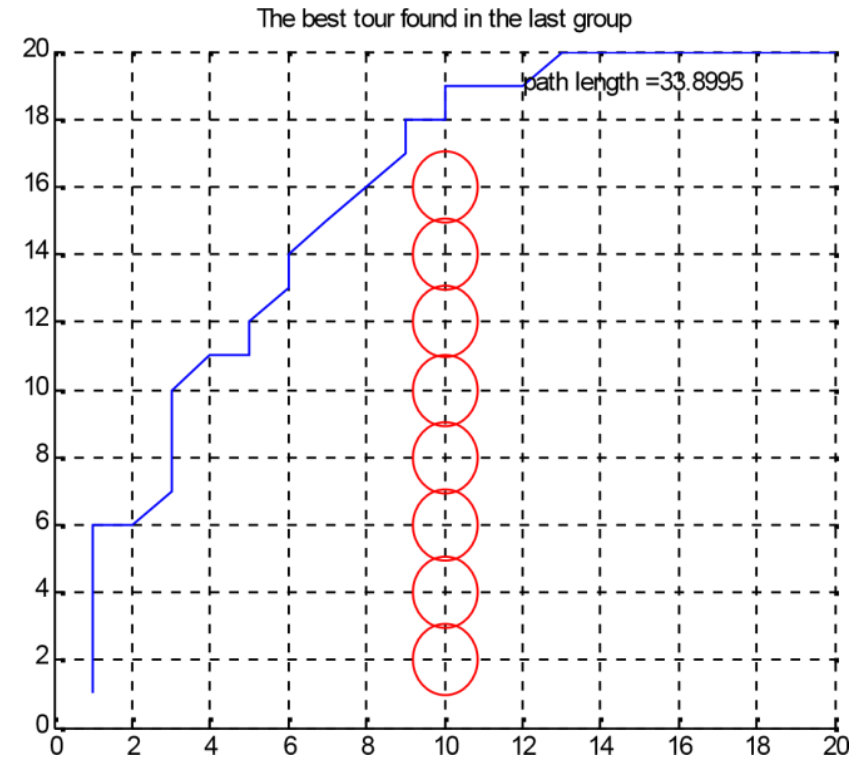

Fig- 4 Optimal Path found with SACO for Experiment 2

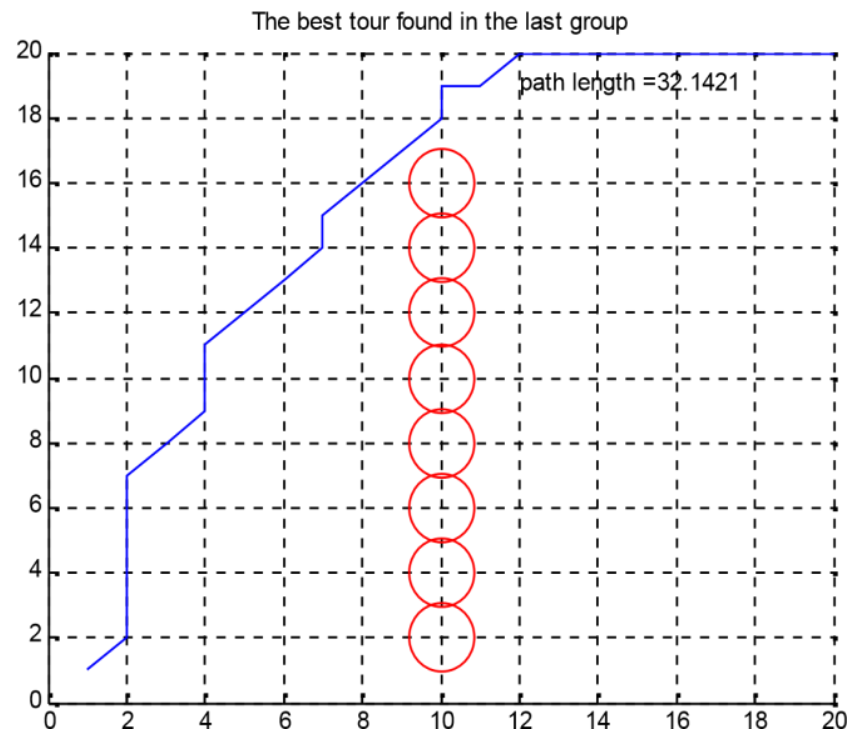

Fig- 5 Optimal Path found with ACO-MH for Experiment 2

\subsection{Experiment 3}

The static obstacles are placed in horizontal line to block the easiest diagonal path. The proposed ACO-MH algorithm utilizes the heuristic function to forget the bad decisions which previously taken by the ants (mobile Robots). The obtained results with ACS and ACO-MH algorithms are shown in figure 6 and 7 respectively. For this experiment there is no significant variation in the optimal path distance. Both the ant algorithms follow the path which is similar to each other. Hence the diagonal path was blocked by the obstacles already.

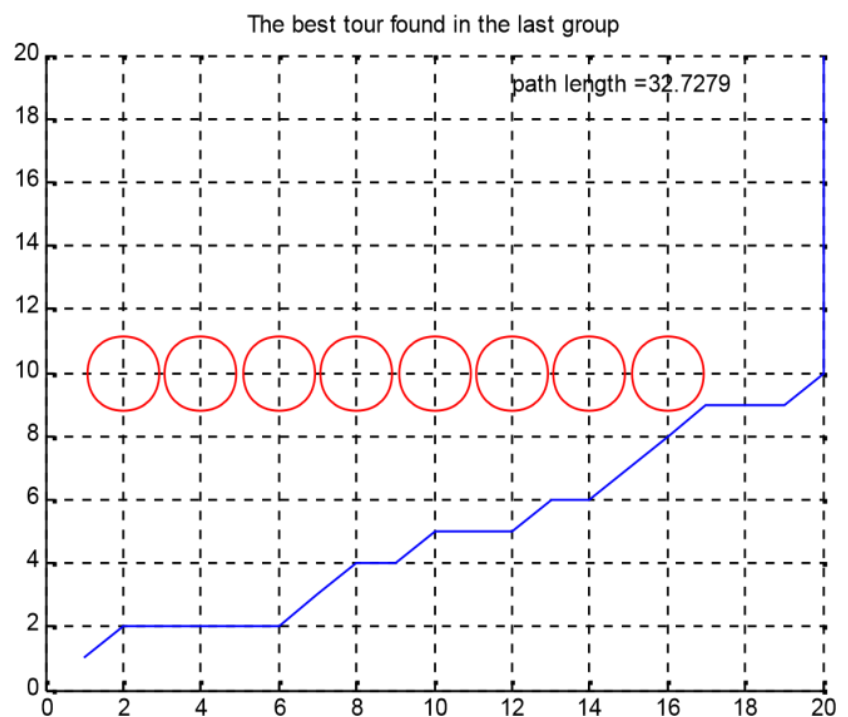

Fig- 6 Optimal Path found with SACO for Experiment 3

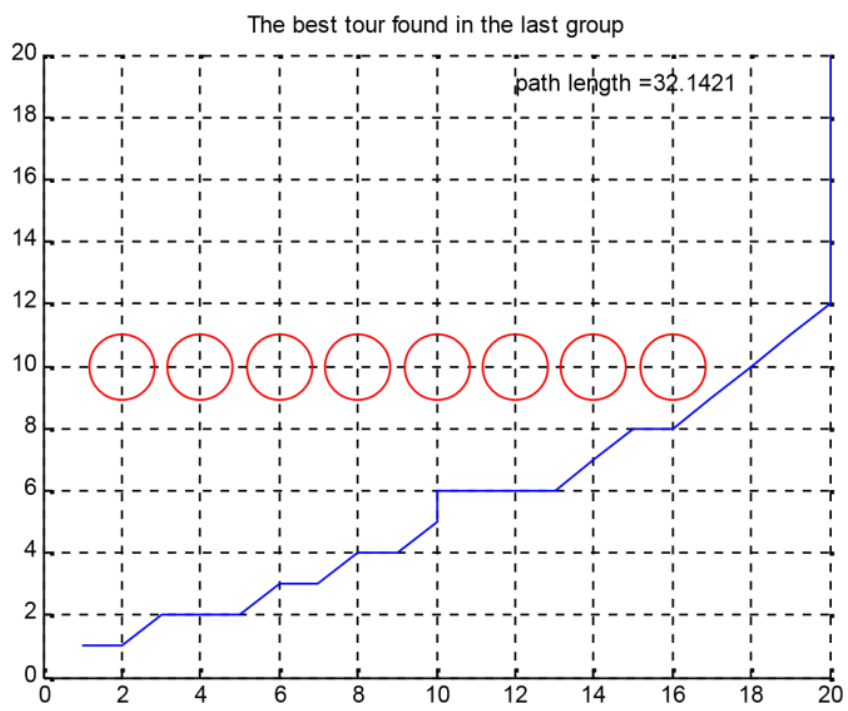

Fig- 7 Optimal Path found with ACO-MH for Experiment3

The Table2 shows the comparison of SACO and ACO-MH algorithms. From the Table 2 the ACO-MH algorithm performs better than SACO in both execution time and path cost.

Table 2 Comparison of Results

\begin{tabular}{|l|l|l|l|l|}
\hline \multirow{2}{*}{ Ex No } & \multicolumn{2}{|l|}{ SACO (ACS) } & \multicolumn{2}{l|}{ ACO -MH } \\
\cline { 2 - 5 } & $\begin{array}{l}\text { Distance } \\
(\mathrm{cm})\end{array}$ & Time $(\mathrm{sec})$ & $\begin{array}{l}\text { Distance } \\
(\mathrm{cm})\end{array}$ & Time (sec) \\
\hline 1 & 31.799 & 3.669818 & 28.6274 & 3.266521 \\
\hline 2 & 33.8995 & 6.568700 & 32.1421 & 6.903165 \\
\hline 3 & 32.7279 & 2.398471 & 32.1421 & 2.369687 \\
\hline
\end{tabular}




\section{CONCLUSIONS}

This paper presents the path planning of mobile robots using the ant colony system algorithm. It reach the target using the ability of the optimization of ant colony algorithm, and control the mobile robot moving to the target (food) from the starting point (nest) in the given static environment. The Number of experiments has been conducted by varying the type of obstacle and number of ants. The output is found to be optimal and satisfying for the given problem with the parameters $\alpha=0.25, \rho=0.1$ and number of ants $m=10$. Further the experiments were extended to change the obstacle shape and location. With increase in complexity of the problem, i.e. with increase in number of obstacles and large number of obstacles exist in the map; this algorithm will require a significant amount of computation time.

\section{REFERENCES}

[1] Abiyev, R., Ibrahim, D., \& Erin, B. (2010). Navigation of mobile robots in the presence of obstacles. Advances in Engineering Software, 41(10), 1179-1186.

[2] Buniyamin, N., Wan Ngah, W. A. J., Sariff, N., \& Mohamad, Z. (2011). A simple local path planning algorithm for autonomous mobile robots. International journal of systems applications, Engineering \& development, 5(2), 151-159.

[3] Cong Y.Z., \& Ponnambalam S.G., (2009). Mobile robot path planning using ant colony optimization. IEEE International Conference on Advanced Intelligent Mechatronics, pp. 851-856

[4] Dorigo, M., \& Gambardella, L. M. (1997). Ant colony system: a cooperative learning approach to the traveling salesman problem. Evolutionary Computation, IEEE Transactions on, 1(1), 53-66.

[5] Dorigo, M., \& Blum, C. (2005). Ant colony optimization theory: A survey. Theoretical computer science, 344(2), 243-278.

[6] M. Dorigo and T. Stutzle (2004), “Ant Colony Optimization", MIT Press, England.

[7] Masehian, E., \& Sedighizadeh, D. (2007). Classic and heuristic approaches in robot motion planning-a chronological review. World Academy of Science, Engineering and Technology, 29(1), 101-106.

[8] Gu, D., \& Hu, H. (2002). Neural predictive control for a car-like mobile robot. Robotics and Autonomous Systems, 39(2), 73-86.

[9] Hu, L., Gu, Z. Q., Huang, J., Yang, Y., \& Song, X. (2008). Research and realization of optimum route planning in vehicle navigation systems based on a hybrid genetic algorithm. Proceedings of the Institution of Mechanical Engineers, Part D: Journal of Automobile Engineering, 222(5), 757-763.

[10] Koren, Y., \& Borenstein, J. (1991, April). Potential field methods and their inherent limitations for mobile robot navigation. Proceedings of IEEE International Conference on In Robotics and Automation, pp. 1398-1404.

[11] Garcia, M. A., Montiel, O., Castillo, O., Sepúlveda, R., \& Melin, P. (2009). Path planning for autonomous mobile robot navigation with ant colony optimization and fuzzy cost function evaluation. Applied Soft Computing, 9(3), pp. 1102-1110.

[12] Sariff, N., \& Buniyamin, N. (2006, June). An overview of autonomous mobile robot path planning algorithms. Proceedings of $4^{\text {th }}$ Student Conference on Research and Development, IEEE,(SCOReD 2006). pp. 183-188.

[13] Sivanandam, S. N., \& Deepa, S. N. (2008). Introduction to Genetic Algorithms. (Vol. 2). Springer.

[14] Soh Chin Yun, Veleppa Ganapathy \& Lim Ooi Chong (2010), "Improved Genetic Algorithms based Optimum Path Planning for Mobile Robot", Proceedings of IEEE $11^{\text {th }}$ International Conference on Control, Automation, Robotics and Vision Singapore, pp. $1565-1570$

[15] Chia, S. H., Su, K. L., Guo, J. H., \& Chung, C. Y. (2010, December). Ant colony system based mobile robot path planning. Proceedings of Fourth International Conference on Genetic and Evolutionary Computing IEEE, (ICGEC) pp. 210213.

[16] Stentz, A. (1994, May). Optimal and efficient path planning for partially-known environments. Proceedings of IEEE International Conference on Robotics and Automation, pp. 3310-3317.

[17] Brand, M., Masuda, M., Wehner, N., \& Yu, X. H. (2010, June). Ant colony optimization algorithm for robot path planning. International Conference on In Computer Design and Applications (ICCDA), 2010 (Vol. 3, pp. V3-436). IEEE.

[18] Dong, J., Liu, B., Peng, K., \& Yin, Y. (2009, May). Robot Obstacle Avoidance based on an Improved Ant Colony Algorithm. WRI Global Congress on Intelligent Systems, 2009. GCIS'09. IEEE. Vol. 3, pp. 103-106).

\section{BIOGRAPHIES}

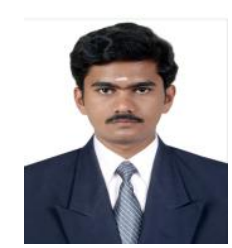

T.Mohanraj graduated from the Anna University, Chennai with a Master Degree in Mechatronics Engineering (Hons.). Currently he is as an Assistant Professor at Kongu Engineering College, Erode. His current research interest is in Condition monitoring, Industrial Automation \& Robotics and Optimization techniques.

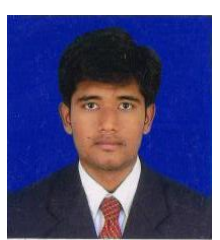

S.Arunkumar graduated from the Anna University, Chennai with a Master Degree in Mechatronics Engineering. He is now an Assistant Professor at Kongu Engineering College, Erode. His current research interest is in MEMS, and Industrial Automation \& Robotics. 


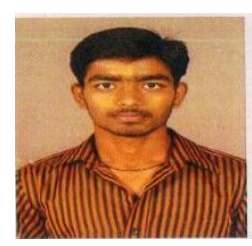

College, Erode.

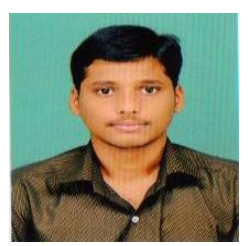

College, Erode.
M.Raghunath graduated from the Anna University, Chennai with a Bachelor Degree in Electrical and Electronics Engineering. He is now pursuing his Master degree in Mechatronics Engineering in Kongu Engineering

M.Anand graduated from the Anna University, Chennai with a Bachelor Degree in Electrical and Electronics Engineering. He is now pursuing his Master degree in Mechatronics Engineering in Kongu Engineering 